\title{
Effect of Simulant Type on the Absorptance and Emittance of Dusted Thermal Control Surfaces in a Simulated Lunar Environment
}

\author{
James R. Gaier ${ }^{1}$ \\ NASA Glenn Research Center, Cleveland OH 44135
}

\begin{abstract}
During the Apollo program the effects of lunar dust on thermal control surfaces was found to be more significant than anticipated, with several systems overheating due to deposition of dust on them. In an effort to reduce risk to future missions, a series of tests has been initiated to characterize the effects of dust on these surfaces, and then to develop technologies to mitigate that risk. Given the variations in albedo across the lunar surface, one variable that may be important is the darkness of the lunar dust, and this study was undertaken to address that concern. Three thermal control surfaces, AZ-93 white paint and AgFEP and AIFEP second surface mirrors were dusted with three different lunar dust simulants in a simulated lunar environment, and their solar absorptivity and thermal emissivity values determined experimentally. The three simulants included JSC-1AF, a darker mare simulant, NU-LHT-1D, a light highlands simulant, and 1:1 mixture of the two. The response of AZ-93 was found to be slightly more pronounced than that of AgFEP. The $\alpha$ increased with fractional dust coverage in both types of samples by a factor of 1.7 to 3.3, depending on the type of thermal control surface and the type of dust. The $\varepsilon$ of the AZ-93 decreased by about 10 percent when fully covered by dust, while that of AgFEP increased by about 10 percent. It was found that $\alpha / \varepsilon$ varied by more than a factor of two depending on the thermal control surface and the darkness of the dust. Given that the darkest simulant used in this study may be significantly lighter than the darkest dust that could be encountered on the lunar surface, it becomes apparent that the performance degradation of thermal control surfaces due to dust on the moon will be strongly dependent on the $\alpha$ and $\varepsilon$ of the dust in the specific locality.
\end{abstract}

\section{Nomenclature}

AgFEP $=0.13 \mathrm{~mm}$ thick fluorinated ethylene propylene (FEP) with a silver reflecting surface on the back

AlFEP $=0.13 \mathrm{~mm}(0.005 \mathrm{in}$.) thick FEP with an aluminum reflecting surface on the back

AxFEP $=$ both AgFEP and AlFEP

AZ-93 = a white thermal control paint formulated by AZ Technologies similar to Z-93

$\alpha=$ absorptivity over the solar spectrum

$\alpha_{\text {rel }} \quad=\alpha / \alpha$ of pristine surface

$\varepsilon \quad=$ emissivity over thermal range (100 to $400 \mathrm{~K})$

$\varepsilon_{\text {rel }} \quad=\varepsilon / \varepsilon$ of pristine surface

$\mathrm{f}=$ fractional of surface covered by dust

\section{Introduction}

A s part of the plans to return humans to the moon, NASA is evaluating the threats that the lunar environment Amay pose to astronaut health and mission success. These threats are numerous and serious, but the one singled out by Apollo astronauts as being the most worrisome is the effects of lunar dust. ${ }^{1}$ One of the most serious system effects was the overheating caused when dust contaminated thermal control surfaces. For example, the batteries on the lunar roving vehicle ran hotter than anticipated, and in fact hotter than their rated operating temperature for part

\footnotetext{
${ }^{1}$ Senior Research Scientist, Space Environment and Experiments Branch, MS 309-2, NASA Glenn Research Center, 21000 Brookpark Road, Cleveland OH 44135.
} 
of the time, even though they were equipped with dust covers and were brushed off at regular intervals. ${ }^{2}$ In anticipation of the much longer stays for lunar surface systems to operate on the lunar surface, a joint task has been developed by NASA's Exploration Technology Development Program's Dust Mitigation Project and Advanced Thermal Control Project to quantify the effects of lunar dust accumulation on thermal control surfaces and to develop mitigation strategies.

Since only a limited amount of fine lunar particles were returned during the Apollo missions, characterization of dust effects has been carried out with lunar simulants. Results reported to date have been limited to measurements of effects of the sub-monolayers of the lunar simulant JSC-1A on the solar absorptivity $(\alpha)$ and thermal emissivity $(\varepsilon)$ of white thermal control paints (AZ-93) and second surface mirrors (AgFEP). It was found that the $\alpha / \varepsilon$ of both types the thermal control surfaces increased by a factor of about 3.5 when fully covered by JSC-1A dust. ${ }^{3}$

But dust on the surface of the moon is not uniform in its thermal optical properties. The lightest crater rays have an $\alpha$ of about 0.50 , and the darkest portions of the mare have an $\alpha$ of about 0.93 . It is reasonable to expect that the effects of the dust on the $\alpha$ and $\varepsilon$ of the dusty thermal control surfaces would depend on the $\alpha$ and $\varepsilon$ of the dust itself. JSC-1A has a measured $\alpha$ of about $0.77,{ }^{4}$ about the same as expected from the average albedo of the soils on the near side lunar surface. ${ }^{5}$ A recently developed lunar simulant designated NU-LHT-1D has a measured $\alpha$ of about $0.51,{ }^{4}$ nearly the same as the lightest soils on the lunar surface. The purpose of this study was to determine what quantitative effect varying the darkness of the dust has on the $\alpha$ and $\varepsilon$ of dusted thermal control surfaces.

\section{Methods and Materials}

This report can be thought of as a follow-on to the report by Gaier. ${ }^{3}$ The methods and materials were only slightly modified from that report. Three lunar simulants were used in the study. The first, JSC-1A, is the same simulant used in the previous study, and in fact all of the data found in this paper using JSC-1A comes from the previous study. The second simulant, NU-LHT-1D, is much lighter in color, and corresponds to a freshly excavated, or highly immature, highlands soil. Soil that is exposed to space weathering is thought to darken as more metallic iron is generated, ${ }^{6}$ so this simulant might be thought of as a light end-member, because all soils found on the lunar surface are expected to be at least this dark. A third simulant was created by the 1:1 mixing of the JSC-1A and the NU-LHT-1D. As expected, it was intermediate in color. However, it would be a mistake to think of this simulant as a mixture of light NU-LHT-1D particles and dark JSC-1 A particles. Both of the root simulants are themselves mixtures of nearly colorless plagioclase and dark pyroxenes, as well as some intermediate color minerals.

Approximately 15 grams of lunar simulant was placed in the activation bowl of the Lunar Dust Adhesion Beljar (LDAB) where it was stirred as a vacuum was pulled down to about $13 \mathrm{~Pa}(100 \mathrm{mTorr})$. At this pressure it was exposed to a radio frequency air plasma for $1 \mathrm{hr}$, with intermittent stirring, to remove organic contaminants. The chamber was then pumped down to about $10^{-4} \mathrm{~Pa}\left(10^{-6} \mathrm{Torr}\right)$ and the simulant was heated at least $16 \mathrm{hr}$ to at least $200{ }^{\circ} \mathrm{C}$, with stirring, to remove water. After cooling to room temperature, the chamber was backfilled with a 5 percent $\mathrm{H}_{2}$ in $\mathrm{He}$ gas to a pressure of $13 \mathrm{~Pa}$ and the dust was exposed to a radio frequency plasma for $1 \mathrm{hr}$, with intermittent stirring, to chemically reduce and to add hydrogen to the dust particle surfaces. This was to mimic the effects of solar wind. The $\mathrm{H}_{2}-\mathrm{He}$ mixture was pumped out and the pressure again dropped to $10^{-4} \mathrm{~Pa}$. Then a recirculating $\mathrm{He}$ refrigerator was used to cool a cold box within the chamber to about $30 \mathrm{~K}$. The resulting condensation of gases onto the surface of the cold box lowered the pressure in the chamber to about $10^{-5} \mathrm{~Pa}$. This was the level of vacuum under which most of the testing was carried out. The dust was transferred from the activation bowl to a 3 in. standard, $38 \mu \mathrm{m}$ stainless steel sieve for application to the samples.

One AZ93 painted aluminum disk, and one aluminum disk with a $0.13 \mathrm{~mm}(5 \mathrm{mil})$ thick FEP appliqué coated either with a few $\mathrm{nm}$ of aluminum or silver with an Inconel ${ }^{\circledR}$ oxidation protection layer applied to the back was mounted in the sample holder for each experimental run. They were suspended in the sample holder by two layers of $0.13 \mathrm{~mm} \mathrm{Kapton}{ }^{\circledR}$, to minimize thermal losses. Temperature was measured by an AWG 36 Type K thermocouple fastened to the back of each sample.

The intensity of the Xe arc lamp was monitored by a photodiode placed directly beneath the lamp. It was found that the intensity of the lamp gradually increased over the first 3 to 5 minutes, and then leveled off and was constant over the remaining time, up to 40 minutes. The total reflectance of several of the AZ-93 samples was measured in either a Lambda 19, a Lambda 950, or a Cary 5000 spectrophotometer over 250 to $2500 \mathrm{~nm}$ wavelengths. The average $\alpha$ calculated from these values, corrected for the air mass zero solar spectrum, was then used in the analysis to calibrate the lamp intensity for each run.

After the lamp intensity stabilized, one of the samples was positioned under the Xe arc lamp. The sample was exposed for $800 \mathrm{sec}$, or until the temperature approached $200{ }^{\circ} \mathrm{C}$, whichever came first. Then the second sample was positioned under the lamp and exposed for a similar duration. Then the lamp was extinguished and the sample 
holder was translated into the cold box, which is lined with an absorbing material (Vel-Black) and maintained at about $30 \mathrm{~K}$ with a recycling helium refrigerator. The samples were allowed to cool until the temperature of both samples was below $20^{\circ} \mathrm{C}$. The sample was then pulled out of the cold box, though still in the vacuum chamber, and allowed to come to near equilibrium temperature before the next test was started.

After heating and cooling data had been collected, the sample was positioned under a sieve filled with activated dust. A Kimball Physics (Wilton, NH) model FRA-2X1-2 electron flood gun sprayed electrons onto the dust as it dropped from the sieve onto the samples. The electron energy was set at $1000 \mathrm{~V}$, and the emission current was about $200 \mu \mathrm{A}$. It should be noted that this was a rather narrow beam, so only some fraction of the falling dust was directly exposed to the electrons. The sample was then translated under the Xe arc lamp, and heated and cooled using the procedure described above for the pristine samples.

Fractional dust coverage was determined from images obtained with an optical microscope at $100 \times$ using image analysis software and a rigorous statistical procedure. The process has been described in detail elsewhere. ${ }^{7}$ The mean of 50 measurements was calculated for each sample, as was the 95 percent confidence interval.

The $\alpha$ and $\varepsilon$ values were derived from thermal modeling which was done using Thermal Desktop (Cullimore \& Ring Technologies) using a model that has been described previously. ${ }^{7}$ Because there were concerns that the intensity of the Xe arc lamp might be changing over time, it was calibrated for each AZ-93 as described above. In the calculation, the lamp intensity was then varied until the calculated heating curve fit the experimental data within 0.5 percent. That lamp intensity was used for the rest of the pristine and dusted heating curves for that sample, and $\alpha$ was varied until the model matched the heating curve data to within 0.5 percent. This process was repeated for each of the AZ-93 samples. This same lamp intensity was also used for the corresponding AgFEP analysis. The data were collected for both types of surfaces at the same time and the AZ-93 data was considered more reliable because of the unknown effects of the light on AgFEP being reflected back up onto the lamp.

\section{Results and Discussion}

\section{A. Effect on White Thermal Control Paint}

White thermal control paints are diffuse reflectors that have relatively higher values of $\alpha$ and higher values of $\varepsilon$. AZ-93, which was used in this study has a solar $\alpha=0.13$ and a thermal $\varepsilon=0.92$. The overall performance of a thermal control surface is dependent on both the $\alpha$ and the $\varepsilon$, and so the ratio $\alpha / \varepsilon$ is typically used, with a smaller value yielding a higher efficiency radiator. The thermal performance of this paint, $\alpha / \varepsilon=0.14$.

The effects of the different simulant types on the relative $\alpha\left(\alpha_{\text {rel }}=\alpha_{\text {dusted }} / \alpha_{\text {pristine }}\right)$ of AZ-93 are shown in Figure 1(a). Although there is a wide scattering in the data, there is an unmistakable trend upwards as the fraction of the surface covered by the dust increases. Least squares fits to the three different populations of data in the figure yielded values for $\alpha_{\text {rel }}$ at full monolayer coverage of 2.2, 2.6, and 3.0 by the lighter, middle, and darker simulants respectively. Previously reported data for the JSC-1AF (darker simulant) have yielded similar values. So it appears that the darkness of the dust does affect the $\alpha$ of a dusted AZ-93 white paint thermal control surface.

The effects of the different simulant types on the relative $\varepsilon\left(\varepsilon_{\text {rel }}=\varepsilon_{\text {dusted }} / \varepsilon_{\text {pristine }}\right)$ of AZ-93 are shown in Figure 1(b). The $\varepsilon_{\text {rel }}$ values are much more tightly grouped, and there is an unmistakable downward trend as the fraction of the AZ-93 surface covered by dust increases. But perhaps surprisingly, the darkness of the dust has no measurable effect, with the least squares lines for all three simulants yielding a $\varepsilon_{\text {rel }}$ of about 0.9 at full dust coverage. This would indicate that despite their different darkness in the visible part of the spectrum, these simulants have similar darkness in the thermal infrared.

Because the effect of dust on the emissivity of AZ-93 is slight, the relative thermal performance, $(\alpha / \varepsilon)_{\text {rel }}$ of the dusted thermal control surfaces is dominated by the $\alpha$. Thus the plot of $(\alpha / \varepsilon)_{\text {rel }}$ as a function of dust coverage (Figure 1(c)) looks very similar to that of the $\alpha_{\text {rel }}$ (Figure 1(a)). Least squares fits to the three different populations of data yielded values for $(\alpha / \varepsilon)_{\text {rel }}$ at full monolayer coverage are summarized in Table I.

Table I. $\alpha / \varepsilon$ Extrapolated to Full Monolayer Coverage

\begin{tabular}{lcccc}
\hline \hline & $\begin{array}{c}\text { Pristine } \\
\alpha / \varepsilon\end{array}$ & $\begin{array}{c}\text { NU-LHT-1D } \\
(\alpha / \varepsilon)_{\text {rel }}\end{array}$ & $\begin{array}{c}1: 1 \\
(\alpha / \varepsilon)_{\text {rel }}\end{array}$ & $\begin{array}{c}\text { JSC-1A } \\
(\alpha / \varepsilon)_{\text {rel }}\end{array}$ \\
\hline AZ93 & 0.14 & 2.6 & 3.0 & 3.4 \\
AgFEP & 0.14 & 1.5 & $2.2^{*}$ & 3.0 \\
\hline \hline
\end{tabular}

*Mixture of three AgFEP and 4 AlFEP samples. 


\section{B. Effects on Second Surface Mirrors}

Unlike white thermal control paints, second surface mirrors are specular reflectors that have lower values of both $\alpha$ and $\varepsilon$ than white paints. AgFEP used in this study has a solar $\alpha=0.10$ and a thermal $\varepsilon=0.70$. The overall performance of a thermal control surface was similar, $\alpha / \varepsilon=0.14$. The JSC-NULHT data set contains 4 AlFEP samples. Although the $\alpha / \varepsilon$ is slightly higher, 0.16 , the relative trends are expected to be the same as the AgFEP, since it is the FEP surface that is exposed to the dust, though this is difficult to verify with such a small number of samples.
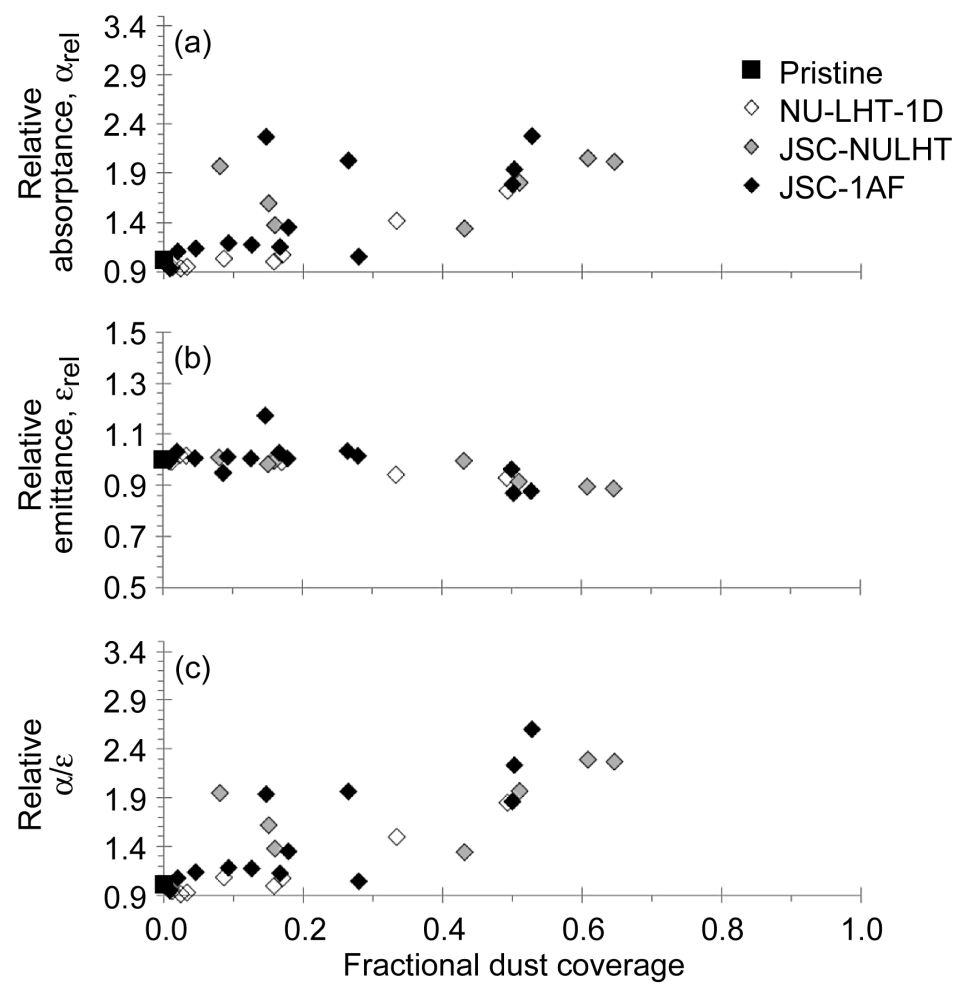

Figure 1. Thermal optical properties of AZ-93 thermal control paint as a function of fractional coverage by three lunar simulant dusts, (a) $\alpha_{\text {rel }}$, (b) $\varepsilon_{\text {rel }}$, and (c) $(\alpha / \varepsilon)_{\text {rel }}$.

The effects of the different simulant types on $\alpha_{\text {rel }}$ of AgFEP are shown in Figure 2(a). Again there is a wide scattering in the data, and again an unmistakable trend upwards as the fraction of the surface covered by the dust increases. Least squares fits to the three different populations of data in the figure yielded values for $\alpha_{\text {rel }}$ at full monolayer coverage of 1.7, 2.3, and 4.2 by the lighter, middle, and darker simulants respectively. It appears that the darkness of the dust affects the $\alpha$ of dusted AgFEP surfaces more strongly than the Z-93 white paint thermal control surface.

The effects of the different simulant types on the $\varepsilon_{\text {rel }}$ of AgFEP are shown in Figure 2(b). Interestingly, the $\varepsilon_{\text {rel }}$ values trend upward as the fraction of the AgFEP surface covered by dust increases, contrary to what was observed in AZ-93. Again, the darkness of the dust has no measurable effect, with the least squares lines for all three simulants yielding a $\varepsilon_{\text {rel }}$ of about 1.1 at full dust coverage. This reinforces the idea that these simulants have similar darkness in the thermal infrared.

Again small dependencies on the effect of dust on the emissivity means that the relative thermal performance, $(\alpha / \varepsilon)_{\text {rel }}$ of the dusted thermal control surfaces is dominated by the $\alpha$. So again the plot of $(\alpha / \varepsilon)_{\text {rel }}$ as a function of dust coverage (Figure 2(c)) looks very similar to that of the $\alpha_{\text {rel }}$ (Figure 2(a)). Least squares fits to the three different populations of data yielded values for $(\alpha / \varepsilon)_{\text {rel }}$ at full monolayer coverage summarized in Table I. So although the $(\alpha / \varepsilon)_{\text {rel }}$ of the pristine AZ-93 and AgFEP are the same, the AgFEP was slightly less affected by the dust. 

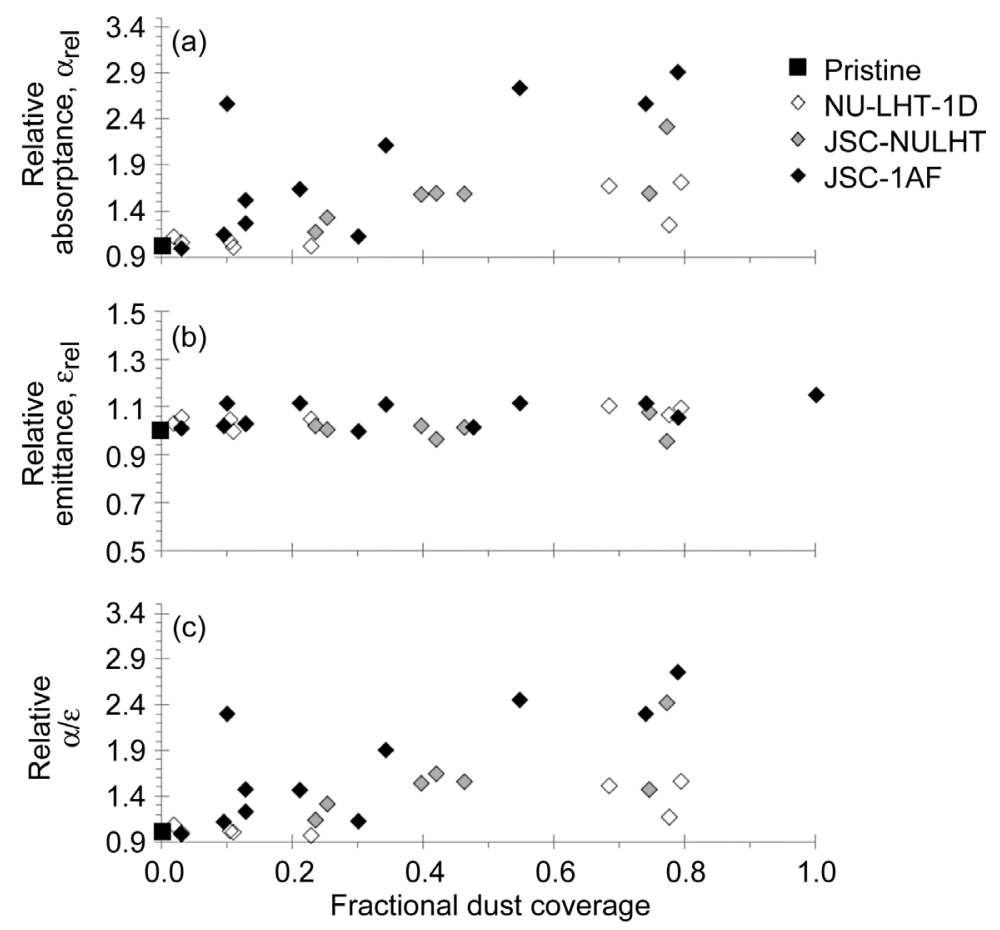

Figure 2. Thermal optical properties of AgFEP second surface mirror thermal control surface as a function of fractional coverage by three lunar simulant dusts, (a) $\alpha_{\text {rel }}$, (b) $\varepsilon_{\text {rel }}$, and (c) $(\alpha / \varepsilon)_{\text {rel }}$.

\section{Rule of Mixtures Calculations of $\alpha$}

A comparison can be made between the measured $\alpha$ and that which might be expected. A reasonable model to use is the rule of mixtures model which assumes that there are no interactions between the dust particles and the thermal control surface. Thus, if a surface is one-quarter covered with dust, the $\alpha$ of that one-quarter should have the $\alpha$ of bulk dust, and the $\alpha$ of the remaining three-quarters of the surface should be unaffected. The definition of $\alpha_{\text {rel }}$ can be written in terms of the rule of mixtures as Equation (1):

$$
\alpha_{\text {rel }}=\frac{\left(f_{\text {dust }} \alpha_{\text {dust }}+\left(1-f_{\text {dust }}\right) \alpha_{\text {pris }}\right)}{\alpha_{\text {pris }}}
$$

Values of $\alpha_{\text {dust }}$ for each of the test dust have been recently calculated from their total reflectance spectra. ${ }^{4}$ Table II shows the comparison between the optical constants as measured spectrophotometrically, and those measured thermally in the LDAB for a thermal control surface that has been fully covered with dust. It can be seen that the rule of mixtures overestimates the effect of the dust on AZ-93 by a factor of 1.8 to 2.0, and on AgFEP by a factor of 2.4 to 3.1 .

Table II. Comparison of the $\alpha_{\text {rel }}$ of Full Dust Covered Thermal Control Surfaces From Optical Measurements Using the Rule of Mixtures Model (Opt) With Those From Thermal Measurements in LDAB (Th)

\begin{tabular}{lcccc}
\hline \hline & AZ-93-Opt & AZ-93-Th & AgFEP-Opt & AgFEP-Th \\
\hline Pristine $\alpha$ & 0.132 & --- & --- & 0.098 \\
NU-LHT-1D & 3.9 & 2.2 & 5.3 & 1.7 \\
NULHT-JSC & 4.9 & 2.6 & 6.5 & 2.3 \\
JSC-1AF & 5.9 & 3.0 & 7.9 & 3.3 \\
\hline
\end{tabular}


This may be due to differences in the optical depth of the dust in the two cases. The spectrophotometric measurement uses a dust layer about $4 \mathrm{~mm}$ thick. This corresponds to 160 layers of $25 \mu \mathrm{m}$ particles. In most cases, the dust layer on the thermal control surfaces was a single layer, and not fully covered. This becomes particularly important because most dust particles are colorless and transparent plagioclase crystals, with a relatively small fraction of dark pyroxene or glass particles. In 160 layers most light will encounter one of the dark particles, but in a single layer most light will not.

\section{Conclusions}

A study of the effects of the darkness of lunar simulants on the $\alpha$ and $\varepsilon$ of dusted thermal control surfaces was undertaken. The response of the white thermal control paint, AZ-93 was found to be slightly more pronounced than that of the second surface mirror thermal control surface, AgFEP or AlFEP. The $\alpha$ increased with fractional dust coverage in both types of samples by a factor of 1.7 to 3.3, depending on the type of thermal control surface and the type of dust. The $\varepsilon$ of the AZ-93 decrease by about 10 percent when fully covered by dust, while that of AgFEP increased by about 10 percent. The thermal performance, $\alpha / \varepsilon$, each thermal control surface covered by a full monolayer for each of the thermal control surfaces by each type of dust varied by a factor of 1.5 to 3.4. So the effect of dust itself varies by more than a factor of two depending on the thermal control surface and the darkness of the dust. Given that the darkest simulant used in this study is probably significantly lighter than the darkest dust that could be encountered on the lunar surface, it becomes apparent that the performance degradation of thermal control surfaces due to dust on the moon will be strongly dependent on the $\alpha$ and $\varepsilon$ of the dust in the specific locality. A simple rule of mixtures calculation overestimates the effect of dust by a factor of 1.8 to 3.1, depending on the surface and the dust. It is suggested that this is due to the compositional heterogeneity of the dust, with the particle number being dominated by particles with low $\alpha$, but the optical properties dominated by the relatively few high $\alpha$ particles.

\section{Acknowledgements}

This work is the result of a great deal of effort by a sizable team of dedicated professionals from many organizations, all of whom the author would like to thank. E. A. Sechkar (Arctic Slope Regional Corporation) and F.P. Lam (Jacobs Sverdrup) provided engineering and technical support of the LDAB facility. S.R. Panko (Arctic Slope Regional Corporation) provided both electrical engineering and test engineering support for the LDAB. The thermal modeling was a team effort involving several people from the NASA Glenn Research Center (GRC) Thermal Systems Branch. D.A. (GRC) oversaw the fabrication of the test samples and acted as a general resource on the behavior of thermal control coatings. Technical, programmatic, and financial support was provided by the Exploration Technology Development Program's Dust Mitigation Project (M.J. Hyatt from GRC) and Advanced Thermal Control Project (R.J. Stephan from the NASA Johnson Space Center).

\section{References}

\footnotetext{
${ }^{1}$ Gaier, J.R., "The Effect of Lunar Dust on EVA Systems During the Apollo Missions," NASA Technical Memorandum, NASA/TM-2005-213610/REV1, (2007).

${ }^{2}$ Gaier, J.R. and Jaworske, D.A., "Lunar Dust on Heat Rejection System Surfaces: Problems and Prospects," Space Technologies and Applications International Forum 2007.

${ }^{3}$ Gaier, J.R., "Effect of Illumination Angle on the Performance of Dusted Thermal Control Surfaces in a Simulated Lunar Environment," International Conference on Environmental Systems paper 2009-01-2420 (SAE, 2009).

${ }^{4}$ Gaier, J.R., and Gustafson, R.J., "Measurement of the Solar Absorptivity and Thermal Emissivity of Lunar Simulants," International Conference on Environmental Systems, AIAA 2010 (to be published).

${ }^{5}$ NASA SP-315, “Apollo 16 Preliminary Science Report,” Chapters 6 and 19, (NASA, 1972).

${ }^{6}$ Noble, S.K., et al., "The Optical Properties of the Finest Fraction of Lunar Soil: Implications for Space Weathering," Meteoritics \& Planetary Science Vol. 36, 2001, pp. 31-42.

${ }^{7}$ Gaier, J.R., Siamidis, J., and Larkin, E.M.G., "Extraction of Thermal Performance Values from Samples in the Lunar Dust Adhesion Bell Jar," $25^{\text {th }}$ Space Simulation Conference, CP-2008-214164, NASA, 2008.
} 